\title{
Development of a Passive Ankle Foot Prosthesis with Manually Adjustable Ankle Stiffness
}

\author{
Tafadzwa Dongo ${ }^{*}$, Benard W. Ikua ${ }^{2}$, Daniel Nyamongo Sagwe ${ }^{3}$, Elliot J. Rouse ${ }^{4}$ \\ ${ }^{1}$ Department of Mechanical and Mechatronic Engineering, Institute of Basic Sciences, Technology and Innovation, Pan African \\ University, Nairobi, Kenya \\ ${ }^{2}$ Department of Mechatronic Engineering, Jomo Kenyatta University of Agriculture and Technology, Nairobi, Kenya \\ ${ }^{3}$ Department of Rehabilitation Medicine, Jomo Kenyatta University of Agriculture and Technology, Nairobi, Kenya \\ ${ }^{4}$ Department of Mechanical Engineering, University of Michigan, Ann Arbor, MI, USA \\ Email: *dongotb@gmail.com
}

How to cite this paper: Dongo, T., Ikua, B.W., Sagwe, D.N. and Rouse, E.J. (2019) Development of a Passive Ankle Foot Prosthesis with Manually Adjustable Ankle Stiffness. Open Journal of Therapy and Rehabilitation, 7, 131-139.

https://doi.org/10.4236/ojtr.2019.74009

Received: September 11, 2019

Accepted: October 13, 2019

Published: October 16, 2019

Copyright (C) 2019 by author(s) and Scientific Research Publishing Inc. This work is licensed under the Creative Commons Attribution International License (CC BY 4.0).

http://creativecommons.org/licenses/by/4.0/

\begin{abstract}
Major advancements have been made in the field of prosthetics, but devices remain largely out of reach for the amputee population domiciled in the developing nations, which makes up $80 \%$ of the entire amputee population of the world. The amputees are left to contend with low function prosthetics that do not mimic the behavior of the natural lost limb, with the long-term use of such devices leading to physical injury to the user. This work was aimed at developing a low-cost ankle-foot prosthesis that affords the user the opportunity to manually alter the stiffness of the ankle, as well storing energy in a forefoot section to aid push-off in late stance. In this paper, the design and results of structural analysis performed on critical parts of the prosthesis are presented, as well as the future direction of the work.
\end{abstract}

\section{Keywords}

Passive Prosthesis, Ankle-Foot, Adjustable Stiffness, Toe-Section, Devices

\section{Introduction}

Amputations are mainly as a result of trauma injuries, congenital deformities and also due to infectious diseases, with the risk of amputations being increased by prolonged armed conflict, natural disasters as well as the breakdown of health services and the inability to control the progression of some diseases [1] [2]. According to the World Health Organization (WHO), there are about 30 million amputees that live in developing countries, with $95 \%$ of them having no access 
to prosthetic devices [3].

Current prosthetic foot development does not target the majority of the end users, with approximately $80 \%$ of the amputee population residing in developing countries [3]. Due to the lack of access, many amputees resort to pole and crutch limbs that are not conducive for activities of daily living and lead to complications such as contracture and upper limb dysfunction [4]. Thus, amputees within the developing world face a variety of challenges due to the lack of the prostheses. This includes the limitation to their mobility as well as making it difficult for the amputees to earn a living, and in some cases being turned into beggars for survival [5].

The most common prosthetic foot in the developing world is the solid ankle cushion heel (SACH) foot, which is a passive flat foot prosthesis designed for household and limited community use [6]. The long-term use of such flat foot prostheses leads to physical injuries for the users such as osteoathritis, osteopenia and subsequent osteoposis, due to musculoskeletal imbalances or pathologies [7]. The high cost of better performing prosthetic devices further hinders the accessibility of the devices due to the economic challenges faced within the developing world [5].

Advanced prosthetic technologies have been developed, but this is realistically available for users in developing countries where there are insured consumers, service members and veterans. These designs are not feasible for the developing world because of the cost, as well as due to the required maintenance from skilled technicians, inadequate adaptation to adverse conditions, and the inability to obtain and maintain a reliable power supply [6].

The stiffness of a natural ankle varies according to the task being undertaken, with the natural ankle exhibiting lower quasi-stiffness during controlled dorsiflexion of stair descent than in level ground walking [8]. The SACH, which is commonly used has a fixed ankle stiffness as the ankle is fixed to the foot and has degree of freedom [9], and as such was designed only for limited mobility. Thus, a device whose stiffness properties can be altered will be of great value to the amputees. To this end, Shepherd and Rouse [10], developed a quasi-passive prosthetic foot, whose stiffness can be altered for different mobility tasks, with the use of a control system and motor. The work of Shephered and Rouse is adopted in this paper, with the aim of developing a passive ankle-foot prosthesis, whose stiffness can be adjusted manually, thereby not requiring controllers or motors that increase the cost, as well as the technical requirements for fitting and maintenance of the device. The manual modulation of the ankle stiffness by the amputee is desirable as they can adjust to a preferred stiffness for different ambulation tasks. This work addresses the issue of low access to prosthetic devices within the developing world, by providing a solution that is aimed at the majority of the amputee population.

\section{Materials and Methods}

The proposed prosthesis has a manual adjustment mechanism for the ankle 
stiffness as well as encompassing a forefoot section. The aim is to make a less complex but functional ankle-foot prosthesis that mimics the functionality of the lost limb. The following were the design requirements for the prosthesis:

1) Light weight such that the weight of the prosthesis does not surpass that of the natural limb.

2) Closely mimicking the dimensions and structure of the natural limb.

3) Incorporation of a fully mechanical adjustment mechanism for the adjustment of the ankle stiffness.

4) Ability to store energy in the forefoot such that it can aid in propelling the leg forward in terminal stance.

The design is presented in three parts. The first and second parts discuss the architecture and development of the adjustment mechanism and forefoot respectively, while the third part presents the complete architecture of the prosthesis. The design and simulation were carried out in SolidWorks ${ }^{\circledR}$.

\subsection{Design of Adjustment Mechanism}

The changing of the ankle stiffness is based on a system of a cam transmission and a leaf spring whose support can be altered [10]. In this current design, the adjustment mechanism is based on a lead screw which is used to move a support slider for the leaf spring. The adjustment is made possible by a radial knob at the back of the foot, which when rotated, will result in the translational motion of the support slider.

Figure 1 shows a schematic of the adjustment mechanism. In this figure, the screw is constrained at both ends in such a way that when rotated, a translational movement of the support attached to it is achieved. The spring is deflected by force Pemanating from movement of the cam transmission that forms the ankle joint. The movement of the support slider causes a change in the stiffness of the spring. This change in the stiffness of the spring will result in a corresponding change in ankle stiffness as described by Shepherd and Rouse [10]. The distance $x$, moved by the slider support upon rotating the screw through an angle $\theta$, is given by

$$
x=l \theta / 2 \pi
$$

where $l$ is the lead. The mechanism is designed to have the maximum travel distance of the support slider as $80 \mathrm{~mm}$. The number of rotations of the screw for

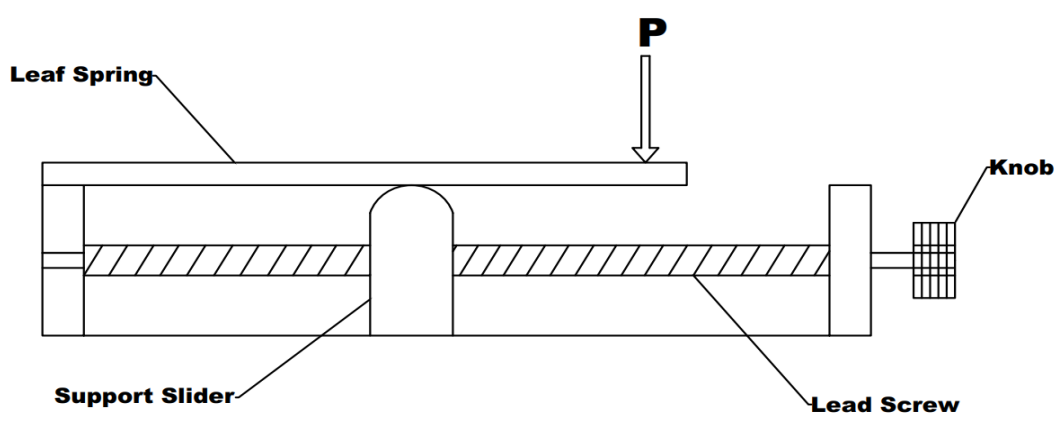

Figure 1. Schematic of adjustment mechanism. 
this travel was arbitrarily taken as 10 , and therefore the lead of the screw was determined as $8 \mathrm{~mm}$. Thus, for a complete revolution of the screw, the slider moves through a distance of $8 \mathrm{~mm}$, as described by the relation in Equation (1).

\subsection{Forefoot Design}

The forefoot section is incorporated in the design of the foot in order to mimic the functionality of the toe section in the natural foot. In normal gait, the foot rocker occurs when the heel is raised from the ground, while the toes remain until the end of the late stance where toe-off occurs [11]. Late stance begins from the moment the heel leaves the ground until the toes also clear the ground. In order to achieve the functionality of the toe section, the design is based on a cantilever attached at the front end of the prosthesis. As such, the forefoot section is modeled as a cantilever beam that deforms (deflection of the beam) and stores energy in the form of strain energy, which is released at toe-off, in order to propel the leg forward.

Figure 2 shows an illustration of the designed prosthesis as it progresses from midstance to toe-off. As the heel begins to rise, the foot rocker occurs, where the foot rolls over the metatarsophalangeal joint. Since the forefoot is a compliant fixture, it will deform as the heel rise continues, thus storing energy in the forefoot. This energy will be released at toe-off, towards the end of late stance, where the foot completely leaves the ground and begins the swing phase of the gait cycle. The forefoot is to be made from Nylon material, as this material exhibits a high strength to weight ratio, as well as high strain energy density [12]. The material is readily available and easy to machine. Energy stored in the forefoot in the form of strain energy, is given by,

$$
U=F^{2} l^{3} / 6 E I
$$

where $F$ is the ground reaction force at the terminal stance, $l$ is the length of the forefoot beam, $E$ is the modulus of the materialused and $I$ is the moment of inertia.

\subsection{Mechanical Structure of Ankle-Foot Prosthesis}

The full structure of the ankle-foot prosthesis is made up of the forefoot section, the adjustment mechanism and a cam and leaf spring transmission for the ankle

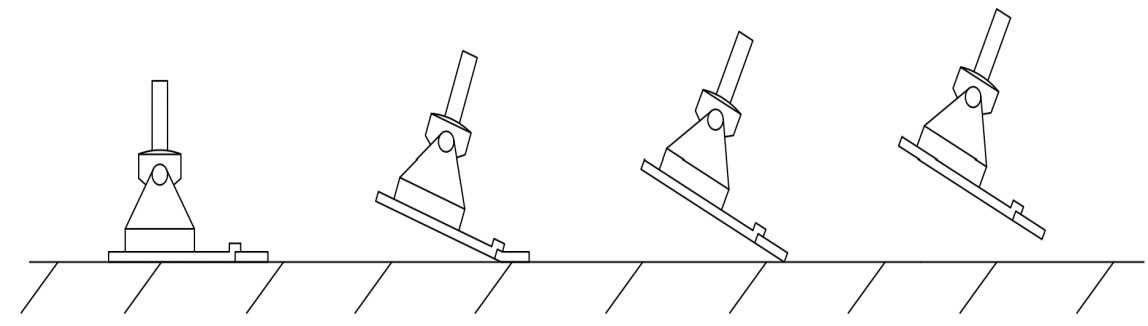

(a)

(b)

(c)

(d)

Figure 2. Illustration of the progression of the foot in late stance. (a) Midstance; (b) Heel rise with toe deflection; (c) Toe-off; (d) Swing. 
joint. The prosthesis has one rotational degree of freedom about the ankle joint, while the forefoot is compliant. The dimensions of the proposed prosthesis are such that they closely resemble the dimensions of a natural ankle-foot complex of a $1.73 \mathrm{~m}$ tall human subject [13]. The dimensions of the natural limb and those of the designed prosthesis are presented in Table 1.

The solid model of the designed prosthesis is shown in Figure 3. Most of the parts of the mechanical structure are made of Aluminium 7075, with the exception of the forefoot, which is made of Nylon for greater flexibility and energy storage.

\section{Results and Discussions}

\subsection{Structural Analysis}

Structural analysis was conducted in order to assess the strength of the structure and to ascertain that the structure is strong enough to withstand the loads to which the prosthesis will be exposed to in use. The analysis was conducted on critical parts of the final model of the prosthesis.

The maximum force, which is achieved at heel strike is used as this is the maximum force that the foot will be exposed to during use. This heel strike impact force used is equivalent to $110 \%$ of the body weight as per literature [14] [15] [16]. The impact force, $F_{\max }$, was therefore obtained using the conversion,

Table 1. Human foot and prosthesis dimensions.

\begin{tabular}{ccc}
\hline Description & $\begin{array}{c}\text { Human Foot } \\
(\mathrm{m})\end{array}$ & $\begin{array}{c}\text { Prosthesis } \\
(\mathrm{m})\end{array}$ \\
\hline Foot Length & 0.26 & 0.287 \\
Toe Length & 0.09 & 0.05 \\
Horizontal distance from back of foot to ankle joint & 0.05 & 0.055 \\
Vertical distance from bottom of foot to ankle joint & 0.07 & 0.085 \\
\hline
\end{tabular}

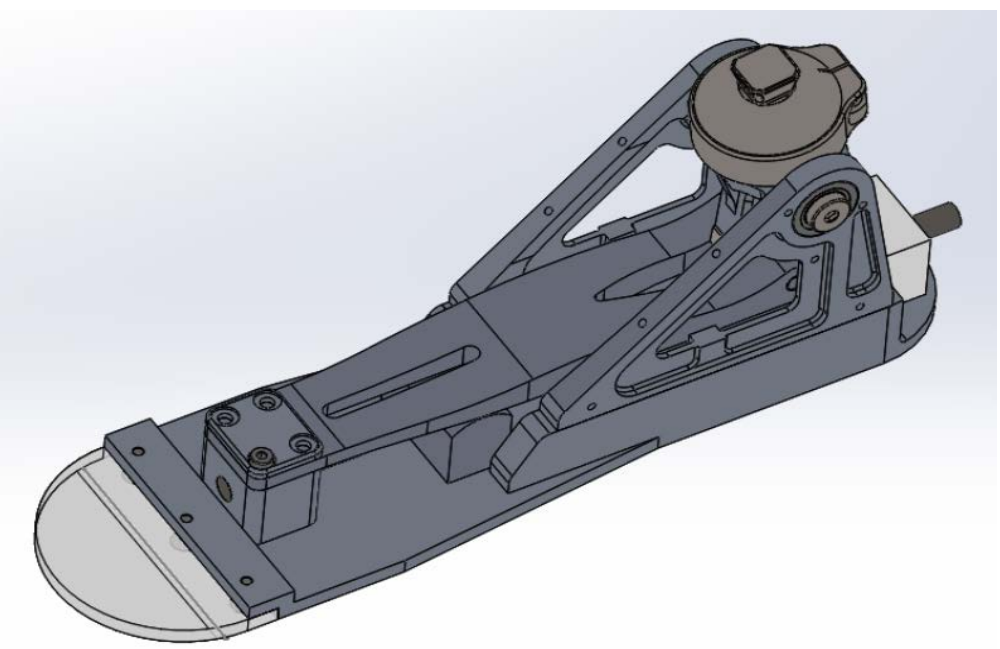

Figure 3. 3D model of the mechanical structure of the designed prosthesis. 


$$
F_{\max }=1.1 \mathrm{mg}
$$

where $m$ is the body weight in $\mathrm{kg}$, and $g$ is acceleration due to gravity, taken as $10 \mathrm{~m} / \mathrm{s}^{2}$. For this study, a body weight of $75 \mathrm{~kg}$ is taken as the design criterion, and as such the value of the vertical Ground Reaction Force (GRF) that the prosthesis would be exposed to on impact with the ground is $825 \mathrm{~N}$. This is the force used in the analysis. Von Mises yield stress criterion was used to analyze the design and the results obtained are presented in Figures 4-7.

Figure 4 shows the stresses of the ankle component based on Von Mises yield strength criterion, as well as the resulting displacement during static loading. The maximum stresses within the part are $4.69 \mathrm{MN} / \mathrm{m}^{2}$, with the yield strength of the material used being $95 \mathrm{MN} / \mathrm{m}^{2}$, thus giving a safety factor of 20 . The maximum deformation of the part is seen to be $1.593 \mu \mathrm{m}$, which for practical purposes, is negligible.

For the foot base, as shown in Figure 5, the maximum stress was found to be $0.79 \mathrm{MN} / \mathrm{m}^{2}$, resulting in a safety factor of 119 . There is no significant deformation within the part, as the maximum deformation is $16.66 \mu \mathrm{m}$.

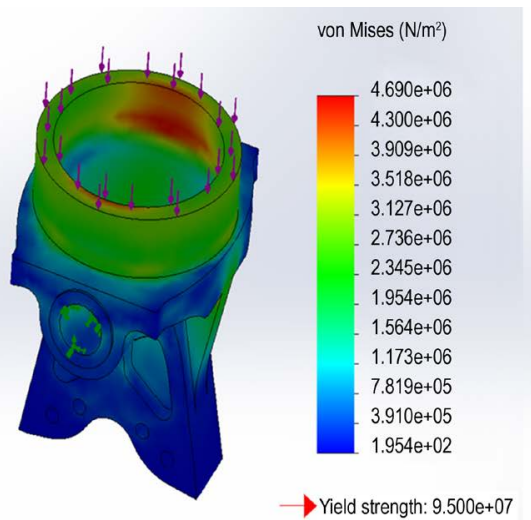

(a)

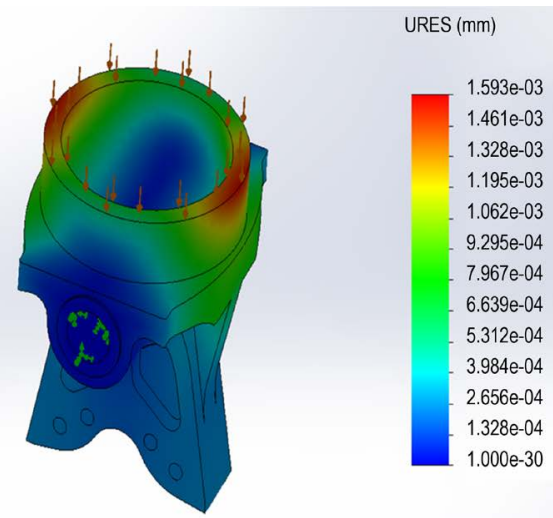

(b)

Figure 4. Stress distribution and resulting deformation for the base of the ankle part. (a) Stress distribution; (b) Deformation.

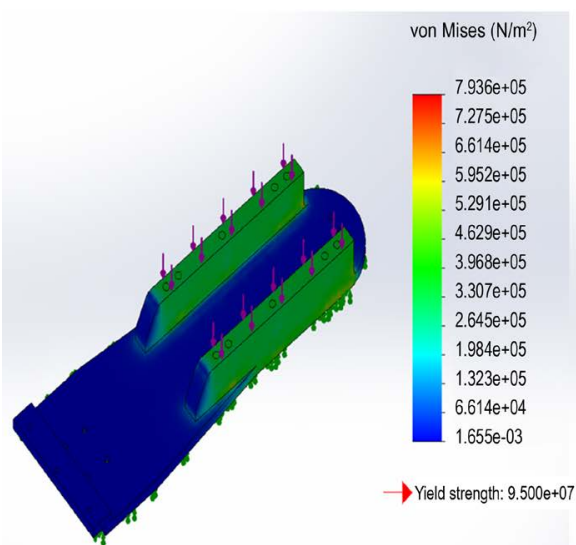

(a)

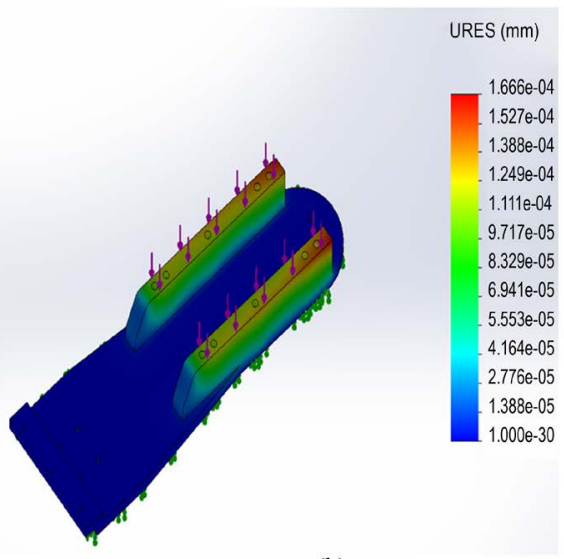

(b)

Figure 5. Stress distribution and resulting deformation for the base of the foot. (a) Stress distribution; (b) Deformation. 


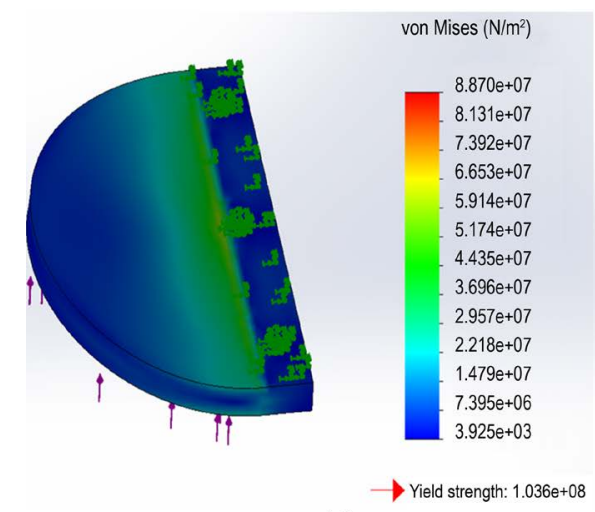

(a)

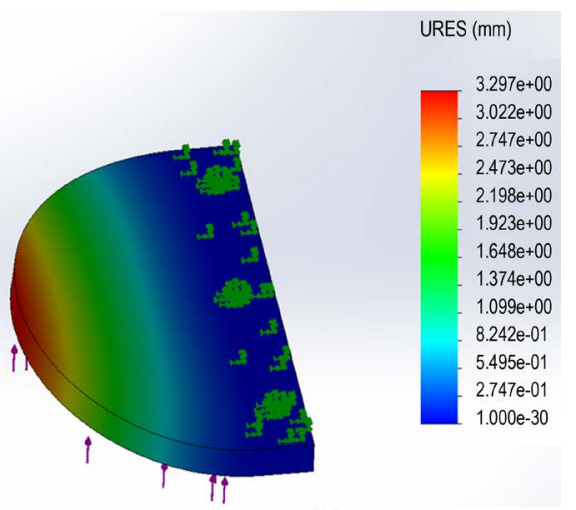

(b)

Figure 6. Stress distribution and resulting deformation for the fore foot. (a) Stress distribution; (b) Deformation.

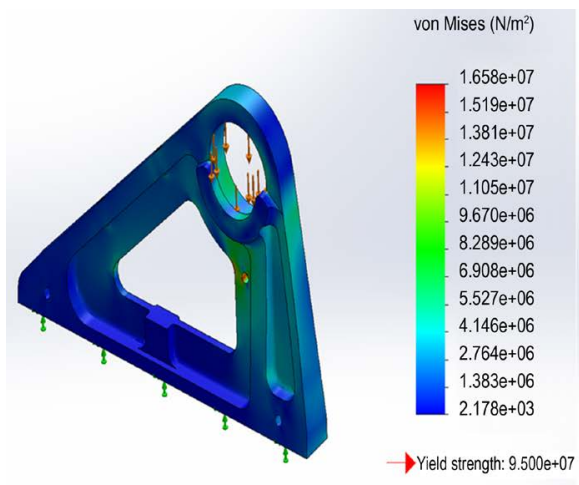

(a)

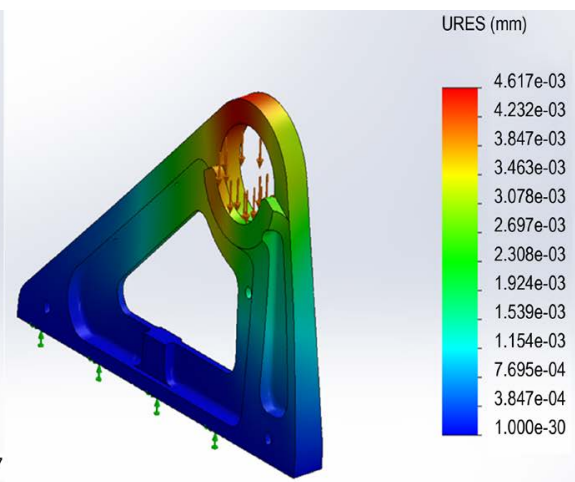

(b)

Figure 7. Stress distribution and resulting deformation for the ankle bracket part. (a) Stress distribution; (b) Deformation.

In the results of the analysis of the forefoot section shown in Figure 6, the maximum stresses are $88.7 \mathrm{MN} / \mathrm{m}^{2}$, as compared to the yield strength of 103.6 $\mathrm{MN} / \mathrm{m}^{2}$ of the material used, resulting in a safety factor of 1.2. The large deformation observed is accepted as the forefoot is designed such that it emulates the flexibility of the toe section in a natural foot. The energy stored during this deformation of the section, as discussed in earlier sections, will then be released in order to assist in propelling the leg forward.

Another critical part on which analysis was carried out is the ankle bracket. The force used in analysis was half of the GRFs $(412.5 \mathrm{~N})$ as there are two brackets that share the total load of $825 \mathrm{~N}$. The results are shown in Figure 7 above. The maximum stresses of $16.58 \mathrm{MN} / \mathrm{m}^{2}$ within the bracket are significantly lower than the yield strength of the material, which is $95 \mathrm{MN} / \mathrm{m}^{2}$, resulting in a safety factor of 5.7. This shows that the brackets are able to withstand the loads that they are exposed to during use. The maximum deformation is $4.617 \mu \mathrm{m}$, which is an insignificant.

\subsection{Properties of the Prosthesis}

The designed prosthesis was also evaluated for other properties, using the work 
by Zaier and Al-Yahmedi [13] as a standard for the natural human foot properties. The designed prosthesis closely resembles the dimensions of the natural ankle-foot complex. This is desirable as the prosthesis would mirror the missing limb. Another property which was evaluated is the weight of the prosthesis. The achieved weight, with all appropriate materials applied in the 3D model, was found to be just above $1.1 \mathrm{~kg}$. For the natural foot, the weight is taken to be $1.37 \%$ of the body weight [13], and thus applying the $75 \mathrm{~kg}$ as before, the comparable weight of an intact ankle-foot complex would be about $1 \mathrm{~kg}$. Thus, the prosthesis also closely mimics the weight of the natural limb.

\section{Conclusions}

In this paper, the design of a passive ankle foot prosthesis was presented. The designed parameters of the prosthesis closely resemble the parameters of an intact human ankle-foot complex. The prosthesis incorporates a forefoot section that can store and release energy in late stance, as well as offer the wearer the ability to alter the stiffness of the prosthesis easily. Structural analysis was conducted on critical parts of the prosthesis and it was shown that the design can withstand the ground reaction forces that the prosthesis would be exposed to in use.

This design is part of an on-going research and the prototype of the prosthesis is being fabricated, which will be experimentally characterized. The main aim is to provide a low-cost ankle foot prosthesis that can closely mimic the functionality of the missing limb. A prosthesis that allows for more functionality and mimics the lost limb as compared to the commonly used prosthetic feet within the developing world, ensures the restoration of ambulation capabilities as well as confidence for the prosthesis users.

\section{Acknowledgements}

This work was sponsored by the African Union through the Pan African University Institute for Basic Sciences, Technology and Innovation (PAUSTI) Research Grant. T.D. thanks Elliot J. Rouse for helping with concept development for this work.

\section{Conflicts of Interest}

There is no conflict of interest regarding the publication of this paper.

\section{References}

[1] Thanni, L.O.A. and Tade, A.O. (2007) Extremity Amputation in Nigeria-A Review of Indications and Mortality. The Surgeon, 5, 213-217. https://doi.org/10.1016/S1479-666X(07)80006-0

[2] Meade, P. and Mirocha, J. (2000) Civilian Landmine Injuries in Sri Lanka. Journal of Trauma and Acute Care Surgery, 48, 735-739. https://doi.org/10.1097/00005373-200004000-00024

[3] Jensen, J.S., Nilsen, R., Zeffer, J., Fisk, J. and Hartz, C. (2006) Clinical Field Testing 
of Vulcanized Rubber Feet for Trans-Tibial Amputees in Tropical Low-Income Countries. Prosthetics and Orthotics International, 30, 195-212. https://doi.org/10.1080/03093640600794692

[4] Ikeda, A.J., Grabowski, A.M., Lindsley, A., Sadeghi-Demneh, E. and Reisinger, K.D. (2014) A Scoping Literature Review of the Provision of Orthoses and Prostheses in Resource-Limited Environments 2000-2010. Part Two: Research and Outcomes. Prosthetics and Orthotics International, 38, 343-362.

https://doi.org/10.1177/0309364613490443

[5] Laferrier, J.Z., Groff, A., Hale, S. and Sprunger, N.A. (2018) A Review of Commonly Used Prosthetic Feet for Developing Countries: A Call for Research and Development. Journal of Novel Physiotherapies, 8, 380.

https://doi.org/10.4172/2165-7025.1000380

[6] Pearlman, J., Cooper, R.A., Krizack, M., Lindsley, A., Wu, Y., Reisinger, K.D., Noon, J., et al. (2008) Lower-Limb Prostheses and Wheelchairs in Low-Income Countries [An Overview]. IEEE Engineering in Medicine and Biology Magazine, 27, 12-22. https://doi.org/10.1109/EMB.2007.907372

[7] Weerakkody, T.H., Lalitharatne, T.D. and Gopura, R.A.R.C. (2017) Adaptive Foot in Lower-Limb Prostheses. Journal of Robotics, 2017, Article ID: 9618375. https://doi.org/10.1155/2017/9618375

[8] Bovi, G., Rabuffetti, M., Mazzoleni, P. and Ferrarin, M. (2011) A Multiple-Task Gait Analysis Approach: Kinematic, Kinetic and EMG Reference Data for Healthy Young and Adult Subjects. Gait \& Posture, 33, 6-13. https://doi.org/10.1016/j.gaitpost.2010.08.009

[9] NZALS (2018) Solid Ankle Cushion Heel (SACH) Foot-Passive Keel. https://www.nzals.co.nz/products/categories/feet-and-ankles/solid-ankle-cushion-h eel-sach-foot-passive-keel

[10] Shepherd, M.K. and Rouse, E.J. (2017) The VSPA Foot: A Quasi-Passive Ankle-Foot Prosthesis with Continuously Variable Stiffness. IEEE Transactions on Neural Systems and Rehabilitation Engineering, 25, 2375-2386. https://doi.org/10.1109/TNSRE.2017.2750113

[11] Perry, J. (2008) Normal Gait. AAOS Atlas of Orthoses and Assistive Devices. E-Book, 61.

[12] Prost, V. (2017) Experimental Validation of the Lower Leg Trajectory Error, an Optimization Metric for Prosthetic Feet. Ph.D. Thesis, Massachusetts Institute of Technology, Cambridge, MA.

[13] Zaier, R. and Al-Yahmedi, A. (2017) Design of Biomechanical Legs with a Passive Toe Joint for Enhanced Human-Like Walking. The Journal of Engineering Research, 14, 166-181. https://doi.org/10.24200/tjer.vol14iss2pp166-181

[14] Mulder, I.A., Holtslag, H.R., Beersma, L.F. and Koopman, B.F. (2014) Keep Moving Forward: A New Energy Returning Prosthetic Device with Low Installation Height after Syme or Pirogoff Amputation. Prosthetics and Orthotics International, 38, 12-20. https://doi.org/10.1177/0309364613485112

[15] AlGheshyan, F.N. (2012) Comparison of Ground Reaction Force in Treadmill Walking and in Overground Walking. University of Miami, Miami, FL.

[16] Barela, A.M., Freitas, P.B.D., Celestino, M.L., Camargo, M.R. and Barela, J.A. (2014) Ground Reaction Forces during Level Ground Walking with Body Weight Unloading. Brazilian Journal of Physical Therapy, 18, 572-579.

https://doi.org/10.1590/bjpt-rbf.2014.0058 